\title{
Analysis of the factors affecting the accuracy of detection for single base alterations by oligonucleotide microarray
}

\author{
Sanzhen $\mathrm{Liu}^{2 *}, \mathrm{Yao}^{\mathrm{Li}^{1 *}}$, Xuping $\mathrm{Fu}^{1}$, \\ Minyan Qiu ${ }^{2}$, Bin Jiang ${ }^{1}$, Hai $\mathbf{W u}^{1}$, \\ Rongyu $\mathrm{Li}^{2}$, Yumin $\mathrm{Mao}^{1}$ and $\mathrm{Yi} \mathrm{Xie}^{1,3}$ \\ ${ }^{1}$ State Key Laboratory of Genetic Engineering \\ Institute of Genetics, School of Life Science \\ Fudan University, Shanghai 200433, P.R. China \\ ${ }^{2}$ Shanghai BioStar Genechip Inc. \\ Shanghai 200092, P.R. China \\ ${ }^{3}$ Corresponding author: Tel, 86-21-6564 3573; \\ Fax, 86-21-6564 2502; E-mail, ymmao@fudan.edu.cn \\ *These authors contributed equally to this work. \\ Accepted 28 December 2004
}

Abbreviation: SSCP, single-strand conformation polymorphism

\begin{abstract}
The oligonucleotide microarray, a high-throughput polymorphism detection technology, holds great promise for the characterization of complex genetic variance. To achieve greater sensitivity and specificity for it to be an effective platform technology we present results and discuss some of the factors influencing signal intensities and single-mismatch discrimination in array-based mutation/SNP detection. Probes with a series of concentrations were spotted onto the slide in order to find the optimal concentration with the identifiable satisfying signals and the stable ratios between matched and mismatched probes. It was found that under our experimental conditions, when the initial probe concentration is higher than the maximum immobilization capability of the slide $(7.5 \mu \mathrm{M})$, the hybridization signal will be saturated and the ratio between matched and mismatched probes will be more stable than at a lower probe concentration. Considering the cost of probes and the systematic stability, a constant spotting concentration of $10 \mu \mathrm{M}$ was selected. The stability of different types of mismatched oligo-DNA duplexes on the glass surface was also confirmed. The results show that the order of stability of mismatched oligo-DNA duplexes on a glass surface is in general agreement with previous re-
\end{abstract}

ports conducted using liquid and polyacrylamide gel pads. This suggests that the influence of the mismatched base pair on the stability of the duplex in a solid hybridization system is similar to that in the solution hybridization environment.

Keywords: mutation; oligonucleotide array sequence analysis; polymorphism, single nucleotide

\section{Introduction}

In order to achieve rapid analysis of mega-quantities of samples with greater accuracy and sensitivity in mutation/SNP discovery and genotyping, there is a definite need for development of a sensitive oligonucleotide microarray platform technology to meet the challenge of advancements in biological sciences and medicine. Presently, a variety of techniques are available for new mutation/polymorphism identification, such as RFLP, direct sequencing, single-strand conformation polymorphism (SSCP) analysis, chemical or enzymatic mismatch cleavage detection, denaturing gradient gel electrophoresis, denaturing HPLC, TaqMan genotyping, and allele-specific ligation (Shi, 2001; Weiner and Hudson, 2002). Most techniques can be broadly classified as either hybridization-based or enzyme-based. Recent development, called the DNA microarray, is a hybridization-based genotyping technique that offers simultaneous analysis of many polymorphisms (Guo et al., 1994; Lipshutz et al., 1995; Hacia et al., 1996; Drmanac et al., 2000). Perfectly matched sequences hybridize more efficiently to their corresponding oligomers on the array, therefore giving stronger fluorescent signals over mismatched probe-target combinations. The advantage of this method is that a large collection of small PCR products can be scanned in one hybridization. Despite the theoretically high levels of scanning capacity, this application suffers some significant limitations in detecting polymorphisms. Recent experiments using microarrays demonstrated only a $97 \%$ accuracy (Patil et al., 2001; Kirk et al., 2002). Furthermore, high false positive rates of $11-21 \%$ have been observed with this technology, limiting its utility in both SNP and tumor analysis (Halushka et al., 1999). In fact, the precision and the discrimination ability of this technology is dependent upon many factors including array fabrication, stringency of hybridization and strategies for designing probes and so on.

The rationale of oligo microarrays used to detect single nucleotide variance is that a single mismatched base pair can reduce the melting temperature $(\mathrm{Tm})$ 
of the hybrid by as much as $10^{\circ} \mathrm{C}$ when the oligonucleotide is shorter than 20 nucleotides (Wallace et al., 1979). However, thermodynamic studies have reported that not all mismatched base pairs have equal stability. G-T and G-A mismatches have been reported as the most stable duplexes. On the other hand, $G$ to $T$ mutation leading to C-T mismatch is one of the most unstable pairs (Werntges et al., 1986; $\mathrm{Ke}$ and Wartell, 1993). Mismatches C-C due to $\mathrm{G}$ to $C$ mutations have also been reported to significantly destabilize the duplexes (Peyret et al., 1999). The order of stability of oligonucleotide-DNA duplexes with different mismatched bases at the same position as presented by lkuta (lkuta et al., 1987) is (from most to least stable) T-A,A-T $>$ G-T,G-A $>$ A-A,T-T,C-T,C-A. Lee et al. (2004) presented a theoretical model, correlating well with previous experimental findings, which was considered as a guideline for single-point mutation probe design. However, his study was limited to properties in solution. The use of a solid surface such as glass slides, however, would introduce surface effects. Even though independent experiments on a three dimensional surface, such as polyacrylamide gel pads, have already proved the value of this model, other experiments especially conducted on the two dimensional surface are imperative for its validation. Yet so far, whether similar nucleotide behavior is performed on solid surfaces has not been reported. In this paper, we present results in detail on the influence of different types of mismatched base pairs on mutation/SNP discrimination and discuss some other problems involved with the design of oligonucleotide microarrays.

\section{Materials and Methods}

\section{Probe design}

Probes were designed using the software, Primer Premier 5.0, and were then followed by manual checks. Melting temperature $(\mathrm{Tm})$ of all probes calculated by the Equation 1 varied from $48^{\circ} \mathrm{C}$ to $52^{\circ} \mathrm{C}$. The allele-specific extension probes were synthesized by Takara Biotech with a $5^{\prime}$ amino group that facilitated their immobilization on the glass slide. A spacer sequence of $10 \mathrm{~T}$ residues was included at the 5 ' end of the gene-specific sequence. The polymorphic sequence was situated near the center of each hybridization sequence.

$T m=2(T+A)+4(G+C)$

(Equation 1)

G, C, A and T represent the numbers of guanine, cytosine, adenine and thymine bases respectively in the oligonucleotide (Anderson, 1999).

\section{Oligo array fabrication}

The amino modified oligonucleotides were spotted onto aldehyde slides (Shanghai Biostar Genechip, Inc.) using a Cartesian Prosys 5501A (Cartesian Te- chnologies, Irvine, CA) fitted with ChipMaker MicroSpotting Technology (TeleChem International, Sunnyvale, CA). Glass slides with immobilized probes were hydrated for $30 \mathrm{~min}$ in $70 \%$ humidity, and then dried for $30 \mathrm{~min}$ at room temperature. They were further soaked at room temperature in $0.2 \%$ SDS for $10 \mathrm{~min}$ and washed in distilled $\mathrm{H}_{2} \mathrm{O}$ for 10 min twice. The slides were then dried again and ready for use.

\section{The preparation of labeled PCR products}

$25 \mu \mathrm{l}$ of PCR reaction system contained $0.2 \mathrm{mM}$ dNTP, $1.5 \mathrm{mM} \mathrm{MgCl} 2,0.2-0.5 \mathrm{ng} / \mu$ l template, $0.4 \mu \mathrm{M}$ Cy5-labeled primer at 5'-end (Synthesized by Takara Biotech) and $3 \mathrm{U}$ rTaq DNA polymerase (Takara Biotech). The mixture was incubated for $2 \mathrm{~min}$ at $94^{\circ} \mathrm{C}$ and 35 cycles of PCR were performed at $94^{\circ} \mathrm{C}$ for $20 \mathrm{~s}, 60^{\circ} \mathrm{C}$ for $20 \mathrm{~s}$ and $72^{\circ} \mathrm{C}$ for $20 \mathrm{~s}$, followed by a final extension at $72^{\circ} \mathrm{C}$ for $5 \mathrm{~min}$. And then 6 $\mu l$ PCR Cy5-labeled products were used for hybridization.

\section{Hybridization and washing}

Fluorescent mixtures including PCR products were mixed with hybridization solution ( $5 \times$ SSC, $0.2 \%$ SDS), denatured for $5 \mathrm{~min}$ at $95^{\circ} \mathrm{C}$, and followed by incubation on ice immediately. Then denatured mixtures were applied onto the microarray slide under a cover glass and placed in $42^{\circ} \mathrm{C}$ chamber for $1 \mathrm{~h}$. The temperature and the reaction time selected was found to be optimal for specificity and sensitivity of singlenucleotide variance analysis (data not shown). After hybridization, the microarray slide was washed at room temperature with washing solution $(2 \times \mathrm{SSC}$, $0.2 \%$ SDS solution) for $5 \mathrm{~min}$ and then dried at room temperature. Short hybridization time and low fluorescent signals, hybridization and washing conditions were optimized and simplified described above.

\section{Signal detection and data analysis}

The arrays were scanned with ScanArray 4000 (GSI Lumonics, Bellerica, MA). The acquired images were analyzed using GenePix 3.0.5 software (Axon Instruments, Inc). All of the spot signal intensities were recorded after subtracting the background intensity.

\section{Results}

\section{Effect of oligonucleotide concentrations on signal intensity and signal ratios of $\mathrm{matched} / \mathrm{m}$ ismatched probes}

To distinguish the relationship between the initial spotting concentration and the actual oligonucleotide density on the surface, different concentrations of oligonucleotide probes labeled with an amino group at the 5 '-end and a Cy3 dye at the 3 '-end, were printed on the glass surface for immobilization. The immobilization efficiency of oligonucleotide probes 
was quantified by fluorescence scanning before and after washing. The results shown in Figure 1 indicate that the surface density increases with increased oligonucleotide concentration when the initial oligonucleotide concentration is less than $7.5 \mu \mathrm{M}$. The density of immobilized probes remains stable above the initial probe concentration of $7.5 \mu \mathrm{M}$. Several repeats of experiments with different probes yielded the similar results (data not shown). However, the analysis of the relationship between initial probe concentration and immobilized probe density is based on the assumption that fluorescent signals represent the density of immobilized probes on the slide. In order to substantiate this assumption and to determine whether the results of the hybridization experiments are in agreement with the above conclusion, we spotted two probes that had one single-base variance (probe $\mathrm{C}$ and probe $\mathrm{T}$ ) with a series of concentrations from $0.08 \mu \mathrm{M}$ to $30 \mu \mathrm{M}$ on one slide. Each oligonucleotide probe was printed in quintuplicate and the intensities were averaged for each probes after hybridization with labeled PCR products of a heterozygote (TC), and homozygotes (TT) and (CC) respectively. The results shown in Figures $2 \mathrm{~A}$ and $2 \mathrm{~B}$ and

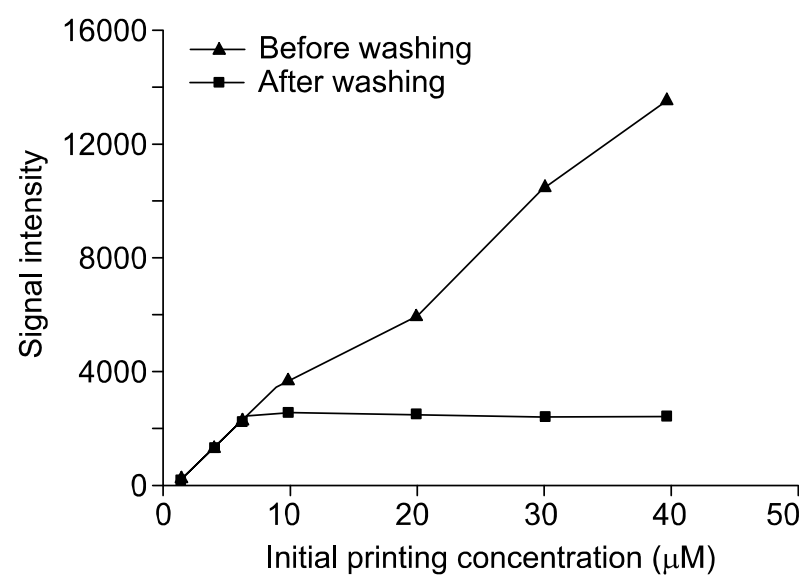

Figure 1. Effect of oligonucleotide probe concentrations on surface density of immobilized oligonuleotides. The spotted oligonucleotide probe was labeled with an amino group at its 5 '-end and Cy3 dye at its 3 '-end, and mixed with an identical sequence oligonucleotide which has an amino group labeled at its $5^{\prime}$-end (ratio of 1:10). Mixtures of oligonucelotide ranging from $2-40 \mu \mathrm{M}$ were spotted on aldehyde glass slides, incubated at room temperature for $30 \mathrm{~min}$ and then washed with $0.2 \%$ SDS.

Table 1. The partial results of hybridization of different types of single base mismatch obtained from the p53 mutation detection.

\begin{tabular}{|c|c|c|c|c|}
\hline Serials & Probes & Referential probes & $\begin{array}{l}\text { Association } \\
\text { tvpe } \text { (liquid-solid) }^{\text {a }}\end{array}$ & $\begin{array}{l}\text { Match } / \text { mismatch } \\
\text { ratio }^{b}\end{array}$ \\
\hline 1 & TACGCCACAAGCTCCA & \multirow{3}{*}{ TACGCCACCAGCTCCA } & G-A & $2.7 \pm 1.4$ \\
\hline 2 & TACGCCACIAGCTCCA & & G-T & $2.9 \pm 1.2$ \\
\hline 3 & TACGCCA $\underline{A} C A G C T C C A$ & & G-A & $2.1 \pm 1.4$ \\
\hline 4 & TACGCCAICAGCTCCA & \multirow{3}{*}{ СТССТСTIGACСTGC } & G-T & $4.3 \pm 1.3$ \\
\hline 5 & СTCСTCTGGACCTGC & & $A-G$ & $7.7 \pm 2.4$ \\
\hline 6 & СTCСTCTGACCTGC & & $A-C$ & $24.1 \pm 4.9$ \\
\hline \multirow{2}{*}{7} & \multirow{2}{*}{ ATGAACCAGAGGCCCA } & \multirow{4}{*}{ ATGAACCGGAGGCCC } & C-A & $8.6 \pm 2.9$ \\
\hline & & & C-T & $9.5 \pm 3.5$ \\
\hline 8 & ATGAACCIGAGGCCCA & & $G_{-T}$ & $20+13$ \\
\hline 9 & ATGAACTGGAGGCCCA & & $c_{-1}^{-1}$ & $122+36$ \\
\hline 10 & 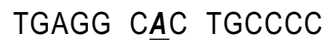 & \multirow{4}{*}{ TGAGG $\underline{\text { CGC }}$ TGCCCC } & $C_{-A}^{-1}$ & $12.2 \pm 3.6$ \\
\hline 11 & TGAGG CIC TGCCCC & & $c-1$ & $20.0 \pm 4.4$ \\
\hline 12 & TGAGG $\underline{I}$ GC TGCCCC & & $a_{-1}$ & $1.9 \pm 1.2$ \\
\hline \multirow{2}{*}{13} & GCACAAACAGGCACCT & & $G-G$ & $1.8 \pm 1.1$ \\
\hline & & \multirow{4}{*}{ GCACAAACACGCACCT } & $G_{-T}$ & $21+12$ \\
\hline 14 & GCACAAACA $\underline{A G C A C C T}$ & & S-1 & \\
\hline 15 & GCACAAACATGCACCT & & $c-A$ & \\
\hline 16 & GCACAAACACACACCT & & C-A & $11.3 \pm 2.6$ \\
\hline
\end{tabular}

${ }^{a}$ Means the associated order of nucleotides used in the table is solid-liquid, e.g, in C-A, C is the nucleotide at an immobilized probe, while $A$ is the nucleotide at a liquid phase probe. ${ }^{b}$ Means the ratio of the signal intensity of reference probes which was perfectly matched with the target to that of mismatched probes 
A

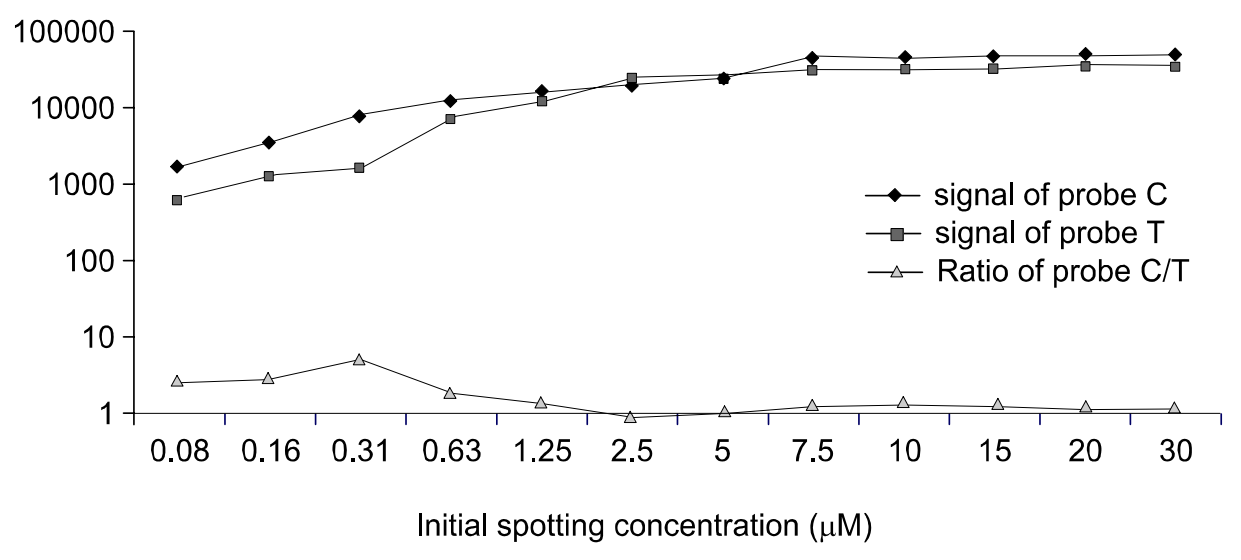

B

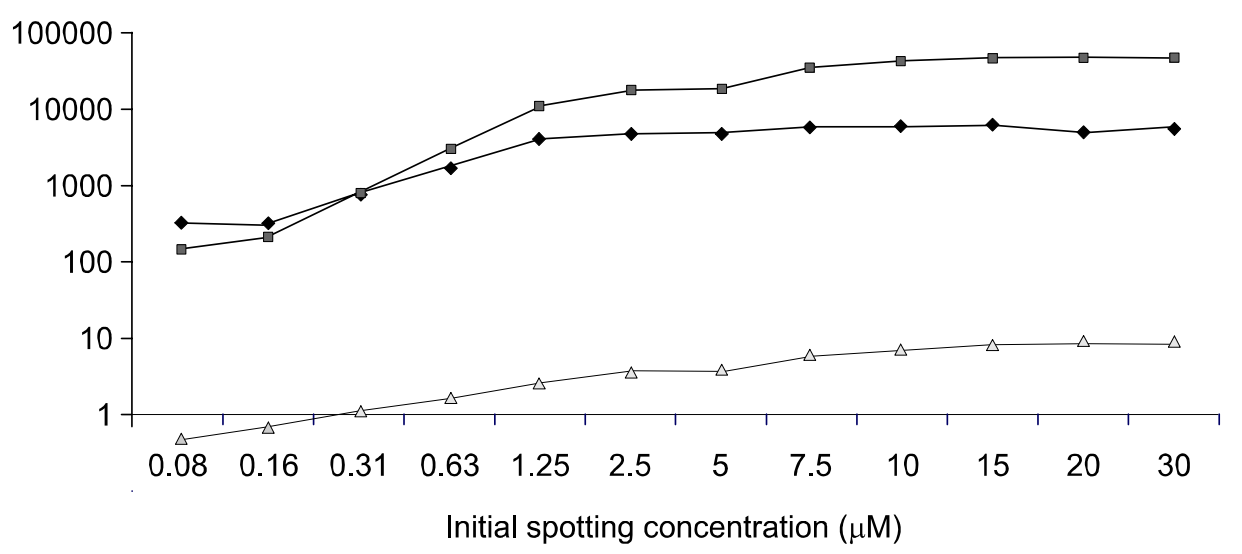

C

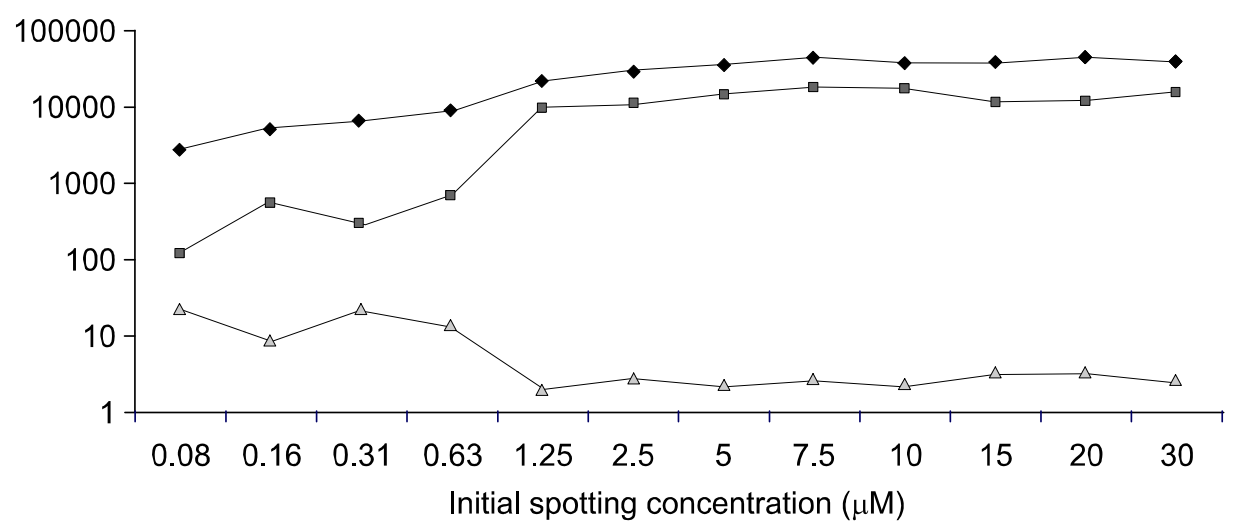

Figure 2. The signal intensities and signal ratios coming from the microarray hybridization. Two different single nucleotide probes (probe C: GTGAAGAACGACCTA AC; probe T: GTGAAGAATGACC TAAC) in series of concentrations from $0.08 \mu \mathrm{M}$ to $30 \mu \mathrm{M}$ were spotted on the same array. Each oligonucleotide probe at a certain concentration was printed in quintuplicate and the intensities were averaged for each probe after hybridization to labeled PCR products with genotypes of CT (Figure 2A), TT (Figure 2B), and CC (Figure $2 C)$ respectively.

$2 \mathrm{C}$ suggest that the hybridization signal intensity is almost saturated and kept stable at concentrations above $5 \mu \mathrm{M}$, which leads to the ratio of two probes at concentrations between $7.5 \mu \mathrm{M}$ and $30 \mu \mathrm{M}$ being more stable than at lower concentrations. Therefore, the concentration itself was not the crucial factor for variance of signal intensities and signal ratios among the certain range of initial probe concentrations (7.5$30 \mu \mathrm{M})$. Under our experimental conditions, the initial concentration of $7.5 \mu \mathrm{M}$ is close to the maximum immobilization capacity of the slide. Considering the cost of probes and the systematic stability, we selected a constant spotting concentration of $10 \mu \mathrm{M}$. 


\section{Effects of the base composition on distinguishability}

Probes in Table 1 were designed to detect hotspot mutations of tumor suppression gene p53 (NC 000017 ) in cancer cells. We designed a mismatched nucleotide close to the center of an oligonucleotide where it has been proven to have a strong destabilizing effect on the duplex. Each oligonucleotide probe was printed in quintuplicate and the intensities were averaged for each probe after hybridization with the labeled PCR product. Match/mismatch ratios in Table 1 are the average of ratios of triplicate experiments. Table 1 lists partial probes' data acquired through hybridization under our experimental conditions.

The kind of mismatched base pair of probe 1 and 3 , resulting from mutation $C$ to $A$, is a $G-A$ mismatch, and that of 2 and 4 , resulting from mutation $C$ to $T$, is a G-T mismatch. The mismatched nucleotide sites on probes 1 and 3 are at the ninth and eighth base positions (from the 5 '-end of the oligonucleotide) respectively. Likewise, there is analogous relationship between probes 2 and 4 . The ratios of perfectly matched probe (Referential Probes) to probe 1, 2, 3, and 4 are $2.7 \pm 1.4,2.9 \pm 1.2,2.1 \pm 1.4,4.3 \pm 1.3$ respectively. Both the differences of ratios between probe 1 and 3 and that of ratios between probe 2 and 4 are not significant. Thus we could conclude that mismatched nucleotide position is a minor factor if located near the center of the oligonucleotide probe as the method we used to design probes.

From the data shown in Table 1 , all match $/ \mathrm{mis}$ match ratios of G-T and G-A mismatches are obviously lower than those of C-T and C-A, which means that G-T and G-A mismatches slightly destabilized a duplex, while C-T and C-A mismatches significantly destabilize it. Our finding resulting from the experiments conducted on glass surface is in general agreement with the Ikuta's reports about the order of stability of oligo-DNA duplex (Ikuta et al., 1987). Accordingly, the influence of the mismatched base pair on the stability of the duplex in the solid hybridization system is similar to that in the solution hybridization environment. In addition, we found that a G-G mismatch, like G-T and G-A, is also slightly destabilizing. The results of various kinds of singlebase mutation probes hybridized to the same fluore- scently labeled target DNA can be seen in Figure 3 . Though different kinds of mismatches give different stabilities of the oligo-DNA duplex, the overall ratio of match/mismatch probe is greater than 1.8 in general (data not shown).

\section{Adding control probe for improving reliability}

In Table 2, primers 1 and 2 are the PCR primers for amplifying the partial fragment of the CYP1A1 gene, Seq $T$ and Seq $C$ are the probes designed to detect its $T$ and $C$ alleles. SeqA is used as a negative control, it has an adenine residue at the mutation site. The results from Figure $4(A), 4(B)$ and $4(C)$, detecting genotypes of TT, TC and CC respectively, whose signal ratios of SeqT/SeqC are $13.5,1.29$ and 0.19 respectively (Table in Figure 4), show that two probes are enough to distinguish single nucleotide polymorphisms. However, under abnormal hybridization conditions such as below the optimal temperature, the ratio of signal intensity of two probes cannot correctly reflect the actual gene polymorphism. From the result shown in Figure 4(D) which is obtained from the hybridization with the PCR of TT genotype, it can be

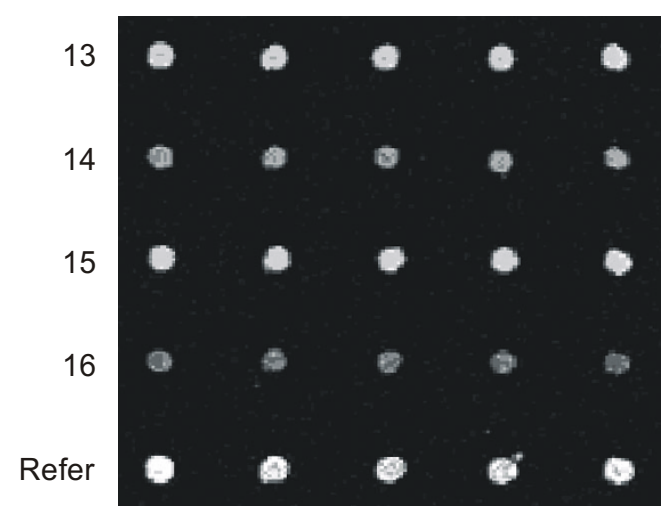

Figure 3. The results of hybridization of various kinds of single-base mutation probes. Probes 13,14,15, 16 and referential probe (Refer) hybridized to the same fluorescently labeled target DNA that is perfectly matched with the referential probe. The sequence of probes is listed correspondingly in Table 1 . The order of signal intensity from high to low represented by pseudo-color is: white $>$ green $>$ blue.

Table 2. The primers and probes of CYP1A1 (NT_024654) for SNP detection.

\begin{tabular}{llcclc}
\hline \multicolumn{1}{c}{ Alias } & \multicolumn{1}{c}{ Sequence 5' to $3^{\prime}$} & Length & Tm & Type & $\begin{array}{c}\text { Nucleotide } \\
\text { variation }\end{array}$ \\
\hline Primer 1 & GAGGTAGCAGTGAAGAGGTGTAGCCG & 26 & - & Forward primer & - \\
Primer 2 & CTGAGGTGGAGATCGTGTGAGC & 24 & - & Reverse primer & - \\
Seq T & CACCTCCTGGGCTCA & 15 & 50.0 & Probe for T allele & T \\
Seq C & CACCTCCEGGGCTC & 14 & 50.0 & Probe for C allele & C \\
Sea A & CACCTCCAGGGCTCA & 15 & 50.0 & Probe as control & A \\
\hline
\end{tabular}




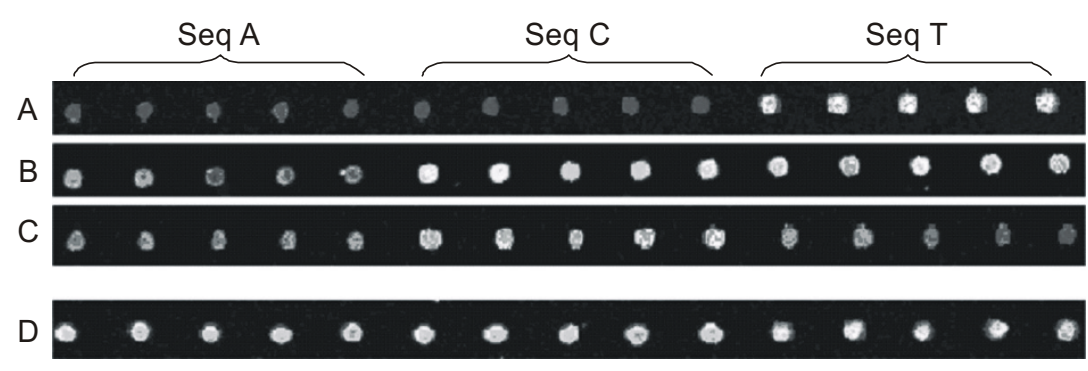

The signal ratios of different probes and the detection results

\begin{tabular}{cccccc}
\hline Figure & $\begin{array}{c}\text { Ratios of } \\
\text { SeaT/SeaC }\end{array}$ & $\begin{array}{c}\text { Ratios of } \\
\text { SeaT/SeaA }\end{array}$ & $\begin{array}{c}\text { Ratios of } \\
\text { SeaC/SeaA }\end{array}$ & $\begin{array}{c}\text { Detection } \\
\text { results }\end{array}$ & Genotype \\
\hline $4(\mathrm{~A})$ & 13.54 & 14.24 & 1.05 & Successful & TT \\
$4(B)$ & 1.29 & 5.08 & 3.93 & Successful & TC \\
$4($ C $)$ & 0.18 & 0.94 & 5.31 & Successful & CC \\
$4(D)$ & 1.35 & 1.33 & 0.99 & Failed & N/A \\
\hline
\end{tabular}

Figure 4. The hybridization results of the fluorescently labeled PCR products of primers 1 and 2. Seq $T, C$ and $A$ indicated on the figure corresponding to those sequences in Table 2, each oligonucleotide probe was printed in quintuplicate. (A), (B) and (C) were the results of the hybridization with the different PCR fragment of the CYP1A1 gene, genotyping of TT, TC and CC respectively. (D) was an abnormal result of the hybridization with the PCR of TT genotype. The signal ratios of different probes and the detection results from $(A),(B),(C)$ and $(D)$ are listed in Table. The order of signal intensity represented by pseudo-colors from high to low is white $>$ red $>$ green $>$ blue.

concluded that it is heterozygous if merely judged by the signal ratio of the Seq T/Seq $C$, about 1.35 (Table in Figure 4). Compared to the signal intensity of the negative control probe (SeqA), SeqC acquired an almost equal signal (the ratio of $S e q C / S e q A$ being 0.99 ) that might be due to nonspecific hybridization. Therefore, without the control probe, the wrong conclusion is inevitable and we cannot even judge whether the result is reliable or not. In order to supervise the hybridization process, designing an additional probe as a negative control is recommended. When at least one of two ratios of test probes to negative control probe is greater than 1.8 , the hybridization experiment is successful. Otherwise, the experiment fails due to non-specific hybridization to the mismatch probe.

\section{Discussion}

The probe density was found to be a controlling factor for the efficiency of target capture as well as for the kinetics of the target/probe hybridization (Southern et al., 1999). A low surface coverage will yield a correspondingly low hybridization signal, and thus decrease the hybridization rate. Accordingly, the intensity of the hybridization signal can differ substantially from one spot to another because of variability of the density of immobile probes on the slide (Southern et al., 1999; Huang et al., 2001; Peterson et al., 2001; Guo et al., 2002). The variance of probe density is derived from many factors, including the properties of the modified slides, spotting solution, printing protocol, post-spotting procedures and the quality of probes. However, it's difficult to accurately control the spotting process, the immobilization efficiency or other factors except for adjusting the initial spotting concentration. Besides the strict control on the procedure of array fabrication, the amount of probe for spotting must be more than the maximum loading capacity of modified slides in order to ensure homogeneous densities of the different probes on the same slide. Under our experiment conditions, the initial concentration of 10 $\mu \mathrm{M}$ for spotting has been proven to be sufficient with aldehyde slides.

Our results in general correlate with previous experimental findings involving the differences in stability of oligo-DNA duplexes with different mismatched bases at the same position. The findings promise to improve the design of oligonucleotide probes for SNP/ mutation detection and save cost on unreasonable design. Generally, there are two alleles presented at one single nucleotide polymorphism locus and two corresponding probes are designed to detect its genotype through comparing signal intensity between them after hybridization. In adopting a two probe design strategy there is the likelihood of achieving high false results as factors affecting the signal in- 
tensity of probe spots are complicated and unpredictable. It is likely that the signal ratios of one probe to another vary significantly under abnormal experimental conditions. The reasonable strategy is to set a negative control probe that has a different base at the mutation site compared to the two original alleles. Considering the identity of the bases, selecting the proper mutated nucleotide in the control probe is essential for discriminating the wild-type homozygous, mutation homozygous, and heterozygous in SNP detection. If it is still difficult to discriminate even when adding a control probe, an additional control probe may be used in order to ensure the accuracy of microarray detection.

\section{Acknowledgement}

We thank Shanghai Biostar Genechip Inc. for manufacturing the microarrays used in this report. Linda Rea and Ben Hazenberg are thanked for their kind help in amending this paper. This work was supported by a grant 2002AA2Z2002 from the National High Technology Research and Development Program of China (863 Program), and by a grant 30371422 from National Natural Science Foundation of China.

\section{References}

Anderson MLM. Nucleic Acid Hybridization, 1999, Oxford: BIOS Scientific Publishers

Drmanac R, Drmanac S, Deane L. Sequencing and fingerprinting DNA by hybridization with oligonucleotide probes. In Meyers, R.A. (ed.), Encyclopedia of Analytical Chemistry. 2000, John Wiley and Sons Ltd, Chichester, UK

Guo Z, Guilfoyle RA, Thiel AJ, Wang R, Smith LM. Direct fluorescence analysis of genetic polymorphisms by hybridization with oligonucleotide arrays on glass supports. Nucleic Acids Res 1994;22:5456-65

Guo Z, Gatterman MS, Hood L, Hansen JA, Petersdorf EW. Oligonucleotide arrays for high-throughput SNPs detection in the MHC class I genes: HLA-B as a model system. Genome Res 2002;12:447-57

Hacia JG, Brody LC, Chee MS, Fodor SP, Collins FS. Detection of heterozygous mutations in BRCA1 using high density oligonucleotide arrays and two-colour fluorescence analysis. Nature Genet 1996;14:441-7

Halushka MK, Fan JB, Bentley K, Hsie L, Shen N, Weder A, Cooper R, Lipshutz R, Chakravarti A. Patterns of singlenucleotide polymorphisms in candidate genes for bloodpressure homeostasis. Nature Genet 1999;22:239-47

Huang E, Satjapipat M, Han SB, Zhou FM. Surface structure and coverage of an oligonucleotide probe tethered onto a gold substrate and its hybridization efficiency for a polynucleotide target. Langmuir 2001;17:1215-24

Ikuta S, Takagi K, Wallace RB, Itakura K. Dissociation kinetics of 19 base paired oligonucleotide-DNA duplexes containing different single mismatched base pairs. Nucleic Acids Res 1987;15:797-811

$\mathrm{Ke} \mathrm{SH}$, Wartell RM. Influence of nearest neighbor sequence on the stability of base pair mismatches in long DNA; determination by temperature-gradient gel electrophoresis. Nucleic Acids Res 1993;21:5137-43

Kirk BW, Feinsod M, Favis R, Kliman RM, Barany F. Single nucleotide polymorphism seeking long term association with complex disease. Nucleic Acids Res 2002;30:3295-311

Lee I, Dombkowski AA, Athey BD. Guidelines for incorporating non-perfectly matched oligonucleotides into targetspecific hybridization probes for a DNA microarray. Nucleic Acids Res 2004;32:681-90

Lipshutz RJ, Morris D, Chee M, Hubbell E, Kozal MJ, Shah $\mathrm{N}$, Shen N, Yang R, Fodor. SP. Using oligonucleotide probe arrays to access genetic diversity. Biotechniques 1995;19: 442-7

Patil N, Berno AJ, Hinds DA, Barrett WA, Doshi JM, Hacker $\mathrm{CR}$, Kautzer CR, Lee DH, Marjoribanks C, McDonough DP, Nguyen BT, Norris MC, Sheehan JB, Shen N, Stern D, Stokowski RP, Thomas DJ, Trulson MO, Vyas KR, Frazer KA, Fodor SP, Cox DR. Blocks of limited haplotype diversity revealed by high-resolution scanning of human chromosome 21. Science 2001;294:1719-23

Peterson AW, Heaton RJ, Georgiadis RM. The effect of surface probe density on DNA hybridization. Nucleic Acids Res 2001;29:5163-8

Peyret N, Seneviratne PA, Allawi HT, SantaLucia JJ. Nearest -neighbor thermodynamics and NMR of DNA sequences with internal A.A, C.C, G.G and T.T mismatches. Biochemistry 1999;38:3468-77

Shi MM. Enabling large-scale pharmacogenetic studies by high-throughput mutation detection and genotyping technologies. Clin Chem 2001;47:164-72

Southern E, Mir K, Shchepinov M. Molecules interactions on microarrays. Nature Genet 1999;21(suppl):S5-9

Wallace RB, Shaffer J, Murphy RF, Bonner J, Hirose T, Itakura K. Hybridization of synthetic oligodeoxyribonucleotides to phi chi 174 DNA: the effect of single base pair mismatch. Nucleic Acids Res 1979;6:3543-57

Weiner MP, Hudson TJ. Introduction to SNPs: Discovery of Markers for Disease. BioTechniques 2002;32:S4-13

Werntges $H$, Steger G, Riesner D, Fritz HJ. Mismatches in DNA double strands: thermodynamic parameters and their correlation to repair efficiencies. Nucleic Acids Res 1986 $14: 3773-90$ 